\title{
Intersections of Nonclassical Unitals and Conics in $\mathrm{PG}\left(2, q^{2}\right)$
}

\author{
Angela Aguglia and Vincenzo Giordano * \\ Dipartimento di Matematica \\ Politecnico di Bari \\ Via G. Amendola 126/B \\ 70126 Bari, Italy \\ a. aguglia@poliba.it, vin.giordano@virgilio.it
}

Submitted: Jun 28, 2009; Accepted: Sep 1, 2010; Published: Sep 13, 2010

Mathematics Subject Classification: E5120, E5121

\begin{abstract}
In $\mathrm{PG}\left(2, q^{2}\right)$ with $q>2$, we determine the possible intersections of a nonclassical Buekenhout-Metz unital $\mathcal{U}$ and a conic passing through the point at infinity of $\mathcal{U}$.
\end{abstract}

\section{Introduction}

The study of the intersection patterns of two relevant geometric objects provides in some cases interesting combinatorial characterizations. An open and rather difficult problem is the classification of all possible intersections between an oval $\Omega$ and a unital $\mathcal{U}$ in a finite projective plane. When $\Omega$ is a conic and $\mathcal{U}$ is a Hermitian or classical unital of $\mathrm{PG}\left(2, q^{2}\right)$, a complete classification of their intersections is given in [1].

In the present paper we make some advances in this direction by considering a suitable family of nonclassical Buekenhout-Metz unitals in $\mathrm{PG}\left(2, q^{2}\right)$, with $q$ any prime power. We will use the following representation of a Buekenhout-Metz unital in $\mathrm{PG}\left(2, q^{2}\right)$ due to Baker and Ebert for $q$ odd and to Ebert for $q$ even. Let $(z, x, y)$ denote homogeneous coordinates for points of $\mathrm{PG}\left(2, q^{2}\right)$. The line $\ell_{\infty}: z=0$ will be taken as the line at infinity, whereas $P_{\infty}$ will denote the point $(0,0,1)$. For $q=2^{h}$, let $C_{0}$ be the additive subgroup of $\operatorname{GF}(q)$ defined by $C_{0}=\{x \in \operatorname{GF}(q): \operatorname{tr}(x)=0\}$, where

$$
\operatorname{tr}:\left\{\begin{array}{l}
\mathrm{GF}(q) \rightarrow \mathrm{GF}(2) \\
x \mapsto x+x^{2}+\ldots+x^{2^{h-1}}
\end{array}\right.
$$

\footnotetext{
${ }^{*}$ Research supported by the Italian Ministry MIUR, Strutture geometriche, combinatoria e loro applicazioni.
} 
is the trace map from $\operatorname{GF}(q)$ onto $\operatorname{GF}(2)$.

Proposition 1.1. (Baker and Ebert[3], Ebert $[4,5]$ ). Let $a, b \in \mathrm{GF}\left(q^{2}\right)$. The point set

$$
\mathcal{U}_{a, b}=\left\{\left(1, t, a t^{2}+b t^{q+1}+r\right) \mid t \in \mathrm{GF}\left(q^{2}\right), r \in \mathrm{GF}(q)\right\} \cup\left\{P_{\infty}\right\}
$$

is a Buekenhout-Metz unital in $\mathrm{PG}\left(2, q^{2}\right)$ if and only if either $q$ is odd and $4 a^{q+1}+\left(b^{q}-b\right)^{2}$ is a nonsquare in $\mathrm{GF}(q)$, or $q$ is even, $b \notin \mathrm{GF}(q)$ and $a^{q+1} /\left(b^{q}+b\right)^{2} \in C_{0}$.

The expression $4 a^{q+1}+\left(b^{q}-b\right)^{2}$, for $q$ odd, or $a^{q+1} /\left(b^{q}+b\right)^{2}$ with $b \notin \operatorname{GF}(q)$, for $q$ even, is the discriminant of the unital $\mathcal{U}_{a, b}$.

Proposition 1.2. (Baker and Ebert[3], Ebert [4, 5]). Every Buekenhout-Metz unital can be expressed as $\mathcal{U}_{a, b}$, for some $a, b \in \mathrm{GF}\left(q^{2}\right)$ which satisfy the discriminant condition of Proposition 1.1. Furthermore, a Buekenhout-Metz unital $\mathcal{U}_{a, b}$ is classical if and only if $a=0$.

Here, using as in [1] the theory of algebraic plane curves, we investigate the intersection patterns of a conic $\Omega$ and a nonclassical Buekenhout-Metz unital $\mathcal{U}_{a, b}$ in $\mathrm{PG}\left(2, q^{2}\right)$ sharing the point at infinity $P_{\infty}$. Our main result is the following theorem.

Theorem 1. In $\mathrm{PG}\left(2, q^{2}\right), q>2$, let $\mathcal{U}_{a, b}$ be a nonclassical Buekenhout-Metz unital and $\Omega$ a conic through the point at infinity $P_{\infty}$ of $\mathcal{U}_{a, b}$. Then the possible intersections between $\mathcal{U}_{a, b}$ and $\Omega$ are the following:

(1) $\mathcal{U}_{a, b}$ and $\Omega$ have only $P_{\infty}$ in common;

(2) $\Omega \cap \mathcal{U}_{a, b}=\Omega, q$ odd;

(3) $\mathcal{U}_{a, b}$ and $\Omega$ have two points in common;

(4) $\mathcal{U}_{a, b} \cap \Omega$ is a Baer subconic of $\Omega$;

(5) $\mathcal{U}_{a, b} \cap \Omega$ is a $k$-arc with $k \in\{q, q+1, q+2\}$ and meets every Baer subconic of $\Omega$ in at most four points;

(6) $\mathcal{U}_{a, b} \cap \Omega$ is the union of two Baer subconics sharing two points or, for $q$ odd, also one point;

(7) $\mathcal{U}_{a, b} \cap \Omega$ is a $k$-arc with $q-6 \sqrt{q}+2 \leqslant k \leqslant q+6 \sqrt{q}+2$ and meets every Baer subconic of $\Omega$ in at most eight points.

Our notation and terminology are standard. For generalities on unitals in projective planes the reader is referred to $[2,6]$; see [7] where background on conics is also found. A recent treatment on algebraic plane curves is [8]. 


\section{Proof of Theorem 1}

We begin by investigating the case in which $\Omega$ is a parabola whose equation is

$$
y=m x^{2}+n x+\ell
$$

where $m \in \operatorname{GF}\left(q^{2}\right)^{*}, n, \ell \in \mathrm{GF}\left(q^{2}\right)$. By Proposition 1.1, a point $P(x, y) \in \Omega$ lies on $\mathcal{U}_{a, b}$ if and only if

$$
(m-a) x^{2}-b x^{q+1}+n x+\ell \in \mathrm{GF}(q) .
$$

Fix a basis $\{1, \epsilon\}$ of $\operatorname{GF}\left(q^{2}\right)$ viewed as a vector space over $\operatorname{GF}(q)$, and write the elements in $\operatorname{GF}\left(q^{2}\right)$ as linear combinations with respect to this basis; that is $z=z_{0}+\epsilon z_{1}$, where $z \in \operatorname{GF}\left(q^{2}\right)$ and $z_{0}, z_{1} \in \operatorname{GF}(q)$. In particular $a=a_{0}+\epsilon a_{1}$ and since $\mathcal{U}_{a, b}$ is a nonclassical Buekenhout-Metz unital, from Proposition 1.2 it follows that $\left(a_{0}, a_{1}\right) \neq(0,0)$.

For $q$ odd, as in [2], choose a primitive element $\beta$ of $\operatorname{GF}\left(q^{2}\right)$ and let $\epsilon=\beta^{(q+1) / 2}$. Then $\epsilon^{q}=-\epsilon$, and $\epsilon^{2}=\omega$ is a primitive element of $\operatorname{GF}(q)$. With this choice of $\epsilon$, condition (1) is equivalent to

$$
\begin{aligned}
g\left(x_{0}, x_{1}\right)= & \left(m_{1}-a_{1}-b_{1}\right) x_{0}^{2}+2\left(m_{0}-a_{0}\right) x_{0} x_{1}+ \\
& +\omega\left(m_{1}-a_{1}+b_{1}\right) x_{1}^{2}+n_{1} x_{0}+n_{0} x_{1}+\ell_{1}=0 .
\end{aligned}
$$

For $q$ even, take an element $\nu$ of $\operatorname{GF}(q)$ such that the polynomial $f(x)=x^{2}+x+\nu$ is irreducible over $\operatorname{GF}(q)$. If $\epsilon \in \operatorname{GF}\left(q^{2}\right)$ is a root of $f(x)$, then $\epsilon^{2}=\epsilon+\nu$ and $\epsilon^{q}=\epsilon+1$. With this choice of $\epsilon$, condition (1) is equivalent to

$$
\begin{aligned}
g\left(x_{0}, x_{1}\right)= & \left(m_{1}+a_{1}+b_{1}\right) x_{0}^{2}+b_{1} x_{0} x_{1}+ \\
& +\left[m_{0}+(\nu+1) m_{1}+a_{0}+(\nu+1) a_{1}+\nu b_{1}\right] x_{1}^{2}+ \\
& +n_{1} x_{0}+\left(n_{0}+n_{1}\right) x_{1}+\ell_{1}=0 .
\end{aligned}
$$

Let $\Gamma$ be the plane algebraic curve with equation $g\left(x_{0}, x_{1}\right)=0$. $\Gamma$ is defined over $\operatorname{GF}(q)$ but it is regarded as a curve over the algebraic closure of $\operatorname{GF}(q)$. The number $N$ of points in $\operatorname{AG}(2, q)$ which lie on $\Gamma$ is the number of points in $\operatorname{AG}\left(2, q^{2}\right)$ on $\mathcal{U}_{a, b} \cap \Omega$.

If $m_{0}=a_{0}, m_{1}=a_{1}, b_{1}=n_{0}=n_{1}=0$ and $\ell_{1} \neq 0$, then $\Omega$ and $\mathcal{U}_{a, b}$ have just $P_{\infty}$ in common.

If $m_{0}=a_{0}, m_{1}=a_{1}$ and $b_{1}=n_{0}=n_{1}=\ell_{1}=0$, then $\Omega$ is entirely contained in $\mathcal{U}_{a, b}$ and $q$ is necessarily odd.

If $m_{0}=a_{0}, m_{1}=a_{1}, b_{1}=0$ and $\left(n_{0}, n_{1}\right) \neq(0,0)$, then $\Gamma$ is a line. In this case $\Gamma$

gives rise to a Baer subconic of $\Omega$ which contains $P_{\infty}$, that is a set of $q+1$ points of $\Omega$ which lie in a Baer subplane of $\operatorname{PG}\left(2, q^{2}\right)$. Actually, with the above setup, lines as well as particular ellipses of $\operatorname{AG}(2, q)$ give rise to a Baer subconic of $\Omega$; see [1][p. 3]. It follows that $\mathcal{U}_{a, b}$ and $\Omega$ share a Baer subconic of $\Omega$.

In the case in which $\Gamma$ is an absolutely irreducible conic, then $\Gamma$ can be either an ellipse, or a parabola, or a hyperbola in $\operatorname{AG}(2, q)$. If $\Gamma$ is an ellipse, $\mathcal{U}_{a, b} \cap \Omega$ is a $(q+2)$-arc; if $\Gamma$ is a parabola, $\mathcal{U}_{a, b} \cap \Omega$ is a $(q+1)$-arc and if $\Gamma$ is a hyperbola, $\mathcal{U}_{a, b} \cap \Omega$ is a $q$-arc. Since two irreducible conics share at most four points, $\mathcal{U}_{a, b} \cap \Omega$ meets every subconic of $\Omega$ in at most four points.

Now, we consider the cases where $\Gamma$ is an absolutely reducible conic, that is: 
(i) $\Gamma$ splits into two distinct nonparallel affine lines each defined over $\operatorname{GF}(q)$, and $\mathcal{U}_{a, b} \cap \Omega$ is the union of two Baer subconics of $\Omega$ sharing two points;

(ii) $\Gamma$ splits into two distinct parallel affine lines defined over $\operatorname{GF}(q)$, and $\mathcal{U}_{a, b} \cap \Omega$ is the union of two Baer subconics of $\Omega$ sharing one point;

(iii) $\Gamma$ is an affine line, counted twice, defined over $\operatorname{GF}(q)$, and $\mathcal{U}_{a, b} \cap \Omega$ is a Baer subconic of $\Omega$;

(iv) $\Gamma$ splits into two distinct nonparallel affine lines defined over $\operatorname{GF}\left(q^{2}\right)$, conjugate to each other, and $\mathcal{U}_{a, b} \cap \Omega$ consists of two points;

(v) $\Gamma$ splits into two distinct parallel affine lines defined over $\operatorname{GF}\left(q^{2}\right)$, conjugate to each other, and $\mathcal{U}_{a, b} \cap \Omega$ consists of one point.

For $q$ odd each of the previous five configurations (i)-(v) occurs and we provide examples for each of these cases:

(i) : $n_{0}=n_{1}=\ell_{1}=0, m_{1}=a_{1}+b_{1}$ and $m_{0} \neq a_{0}$;

(ii) : $m_{1}=a_{1}+b_{1}, b_{1} \neq 0, m_{0}=a_{0}, n_{0}=n_{1}=0, \ell_{1}=-2 \omega b_{1}$;

(iii) $n_{0}=n_{1}=\ell_{1}=0, m_{1}=a_{1}+b_{1}, b_{1} \neq 0, m_{0}=a_{0}$;

(iv) : $b_{1}=0,\left(a_{0}, a_{1}\right) \neq(-1,-1), m_{1}=1+a_{1}, m_{0}=1+a_{0}, n_{0}=n_{1}=\ell_{1}=0$;

(v) : $m_{1}=a_{1}+b_{1}, b_{1} \neq 0, m_{0}=a_{0}, n_{0}=n_{1}=0, \ell_{1}=-2 \omega^{2} b_{1}$.

Let $q$ be even. If two linear components of $\Gamma$ were parallel then $b_{1}$ should be zero, a contradiction. Therefore just the cases (i) and (iv) can occur and we provide examples for these two cases:

(i) $n_{0}=n_{1}=\ell_{1}=0, m_{1}=a_{1}+b_{1}$;

(iv) $n_{0}=n_{1}=\ell_{1}=0, m_{1}=1+a_{1}+b_{1}, m_{0}=\nu, a_{0}=\nu b_{1}^{2}$.

Now, suppose that $\Omega$ is a hyperbola. Since the stabilizer of $\mathcal{U}_{a, b}$ in $P G L\left(3, q^{2}\right)$ acts on the points of $\ell_{\infty} \backslash P_{\infty}$ as a transitive permutation group, we may assume that $\Omega$ has equation

$$
y=\frac{m x+n}{x+\ell}
$$

with $\ell, m, n \in \operatorname{GF}\left(q^{2}\right)$ and $\ell m-n \neq 0$. A point $P(x, y) \in \Omega$ lies on $\mathcal{U}_{a, b}$ if and only if

$$
\frac{m x+n}{x+\ell}-a x^{2}-b x^{q+1} \in \mathrm{GF}(q) \text {. }
$$


Condition (4) can be written as

$$
\begin{aligned}
f\left(x_{0}, x_{1}\right)= & {\left[\left(a_{1}+b_{1}\right) x_{0}^{2}+2 a_{0} x_{0} x_{1}+\omega\left(a_{1}-b_{1}\right) x_{1}^{2}\right]\left(x_{0}^{2}-\omega x_{1}^{2}+\right.} \\
& \left.+2 \ell_{0} x_{0}-2 \omega \ell_{1} x_{1}+\ell_{0}^{2}-\omega \ell_{1}^{2}\right)-m_{1} x_{0}^{2}+m_{1} \omega x_{1}^{2}+ \\
& +\left(\ell_{1} m_{0}-n_{1}-\ell_{0} m_{1}\right) x_{0}+\left(n_{0}+\omega \ell_{1} m_{1}-\ell_{0} m_{0}\right) x_{1} \\
& +\ell_{1} n_{0}-\ell_{0} n_{1}=0,
\end{aligned}
$$

for $q$ odd and

$$
\begin{aligned}
f\left(x_{0}, x_{1}\right)= & {\left[\left(a_{1}+b_{1}\right) x_{0}^{2}+b_{1} x_{0} x_{1}+\left(\left(a_{0}+a_{1}\right)+\nu\left(a_{1}+b_{1}\right)\right) x_{1}^{2}\right]\left(x_{0}^{2}+x_{0} x_{1}+\right.} \\
& \left.+\nu x_{1}^{2}+\ell_{1} x_{0}+\ell_{0} x_{1}+\ell_{0}^{2}+\ell_{0} \ell_{1}+\nu \ell_{1}^{2}\right)+m_{1} x_{0}^{2}+m_{1} x_{0} x_{1}+\nu m_{1} x_{1}^{2}+ \\
& +\left(\ell_{0} m_{1}+\ell_{1} m_{0}+n_{1}\right) x_{0}+\left(\ell_{0} m_{0}+\ell_{0} m_{1}+\nu \ell_{1} m_{1}+n_{0}\right) x_{1}+ \\
& +\ell_{1} n_{0}+\ell_{0} n_{1}=0,
\end{aligned}
$$

for $q$ even.

Let $\Phi$ denote the plane algebraic curve with equation $f\left(x_{0}, x_{1}\right)=0$. $\Phi$ is defined over $\operatorname{GF}(q)$ but it is regarded as a curve over the algebraic closure of $\operatorname{GF}(q)$. Since $\mathcal{U}_{a, b}$ is a Buekenhout-Metz unital the coefficients of the degree-four terms in (5), as well as in (6), do not vanish identically and so $\Phi$ is a plane quartic curve.

Lemma 2. $\Phi$ is an absolutely irreducible curve of $\mathrm{PG}(2, q)$.

Proof. By way of contradiction, we suppose that $\Phi$ is a reducible curve. We are going to determine the points of $\Phi$ at infinity. Let

$$
\phi(x)=\left(a_{1}+b_{1}\right) x^{2}+2 a_{0} x+\omega\left(a_{1}-b_{1}\right)
$$

for $q$ odd, and

$$
\phi(x)=\left(a_{1}+b_{1}\right) x^{2}+b_{1} x+\left(a_{0}+a_{1}\right)+\nu\left(a_{1}+b_{1}\right)
$$

for $q$ even. The polynomial $\phi(x)$ is irreducible over $\operatorname{GF}(q)$. In fact, when $q$ is odd, we have that

$$
4 a_{0}^{2}-4 \omega\left(a_{1}^{2}-b_{1}^{2}\right)=4 a^{q+1}+\left(b^{q}-b\right)^{2}
$$

that is, the discriminant of $\phi(x)=0$ is a nonsquare in $\operatorname{GF}(q)$. For $q$ even, using the additive property of the trace function we get

$$
\begin{aligned}
\operatorname{tr}\left(\frac{\nu a_{1}^{2}+a_{1}^{2}+a_{0} a_{1}+a_{0} b_{1}+b_{1} a_{1}+\nu b_{1}^{2}}{b_{1}^{2}}\right)= & \operatorname{tr}\left(\frac{a_{0}^{2}+a_{0} a_{1}+\nu a_{1}^{2}}{b_{1}^{2}}\right)+ \\
& +\operatorname{tr}\left(\frac{a_{0}^{2}+a_{1}^{2}+a_{0} b_{1}+b_{1} a_{1}}{b_{1}^{2}}\right)+\operatorname{tr}(\nu) .
\end{aligned}
$$

Since

$$
\operatorname{tr}\left(\frac{a_{0}^{2}+a_{0} a_{1}+\nu a_{1}^{2}}{b_{1}^{2}}\right)=\operatorname{tr}\left(\frac{a^{q+1}}{\left(b^{q}+b\right)^{2}}\right)=0,
$$

and

$$
\operatorname{tr}\left(\frac{a_{0}^{2}+a_{1}^{2}+a_{0} b_{1}+b_{1} a_{1}}{b_{1}^{2}}\right)=\operatorname{tr}\left(\frac{a_{0}+a_{1}}{b_{1}}+\left(\frac{a_{0}+a_{1}}{b_{1}}\right)^{2}\right)=0,
$$


it follows that

$$
\operatorname{tr}\left(\frac{\nu a_{1}^{2}+a_{1}^{2}+a_{0} a_{1}+a_{0} b_{1}+b_{1} a_{1}+\nu b_{1}^{2}}{b_{1}^{2}}\right)=1,
$$

and $\phi(x)=0$ has no solutions in $\operatorname{GF}(q)$.

Now, let $i \in \operatorname{GF}\left(q^{2}\right)$ denote a root of $\phi(x)$; then $i$ and $i^{q}$ are both roots of $\phi(x)$ and thus the points of $\Phi$ at infinity are

$$
Q^{+}(0, \epsilon, 1), Q^{-}(0,-\epsilon, 1), P^{+}(0, i, 1), P^{-}\left(0, i^{q}, 1\right)
$$

for $q$ odd and

$$
Q^{+}(0, \epsilon, 1), Q^{-}(0,1+\epsilon, 1), P^{+}(0, i, 1), P^{-}\left(0, i^{q}, 1\right)
$$

for $q$ even.

In both cases, since $a \neq 0$, the points at infinity of $\Phi$ are all distinct; therefore each of these points is simple, and hence they must lie just on one component of $\Phi$.

Let $\mathcal{C}$ be a component of $\Phi$ through $Q^{+}$. Since $\Phi$ is defined over $\operatorname{GF}(q)$ also the image $\mathcal{C}^{q}$ of $\mathcal{C}$ under the Frobenius collineation $(x, y) \rightarrow\left(x^{q}, y^{q}\right)$ is a component of the same degree of $\Phi$ through $Q^{-}$. If $\mathcal{C}$ were an irreducible cubic then $\mathcal{C}^{q}=\mathcal{C}$, that is, $\mathcal{C}$ would be defined over $\operatorname{GF}(q)$. In this case $\Phi$ would split into the cubic $\mathcal{C}$ and a line $\ell$ which is also defined over $\operatorname{GF}(q)$. Both points $Q^{+}$and $Q^{-}$lie on $\mathcal{C}$, but neither $P^{+}$nor $P^{-}$belongs to $\mathcal{C}$; otherwise the cubic $\mathcal{C}$ would contain four points at infinity, which is impossible. Then $\ell$ turns out to be an affine line through $P^{+}$and $P^{-}$, a contradiction. Now we investigate the following cases:

(a) $\Phi$ splits into two distinct irreducible conics defined over $\operatorname{GF}(q)$;

(b) $\Phi$ splits into an irreducible conic defined over $\mathrm{GF}(q)$ and two lines defined over $\mathrm{GF}\left(q^{2}\right)$ and conjugate over $\operatorname{GF}(q)$;

(c) $\Phi$ splits into four distinct lines defined over $\mathrm{GF}\left(q^{2}\right)$ two by two conjugate over $\mathrm{GF}(q)$;

(d) $\Phi$ splits into two distinct absolutely irreducible conics defined over $\operatorname{GF}\left(q^{2}\right)$ and conjugate over $\mathrm{GF}(q)$.

In the first three cases we may assume that $\Phi$ splits into two conics $\mathcal{C}_{1}$ and $\mathcal{C}_{2}$ defined over $\operatorname{GF}(q)$, with $P^{+}$and $P^{-}$on $\mathcal{C}_{1}$ and $Q^{+}$and $Q^{-}$on $\mathcal{C}_{2}$. In case (a), both $\mathcal{C}_{1}$ and $\mathcal{C}_{2}$ are absolutely irreducible; in case (b) one of them is absolutely irreducible whereas the other one is irreducible over $\operatorname{GF}(q)$ but reducible over $\operatorname{GF}\left(q^{2}\right)$; finally, in case (c) both the components $\mathcal{C}_{1}$ and $\mathcal{C}_{2}$ are absolutely reducible over $\operatorname{GF}\left(q^{2}\right)$. We first investigate the case $q$ odd. We may assume that $\mathcal{C}_{1}$ and $\mathcal{C}_{2}$ have equations

$$
\begin{aligned}
& \mathcal{C}_{1}: f_{1}\left(x_{0}, x_{1}\right)=\left(x_{0}-i x_{1}\right)\left(x_{0}-i^{q} x_{1}\right)+\alpha x_{0}+\beta x_{1}+\gamma=0, \\
& \mathcal{C}_{2}: f_{2}\left(x_{0}, x_{1}\right)=\left(x_{0}-\epsilon x_{1}\right)\left(x_{0}+\epsilon x_{1}\right)+\alpha^{\prime} x_{0}+\beta^{\prime} x_{1}+\gamma^{\prime}=0
\end{aligned}
$$

where $\alpha, \alpha^{\prime}, \beta, \beta^{\prime}, \gamma, \gamma^{\prime} \in \mathrm{GF}(q)$ and

$$
\rho f\left(x_{0}, x_{1}\right)=f_{1}\left(x_{0}, x_{1}\right) f_{2}\left(x_{0}, x_{1}\right) .
$$


Comparing in (7) the terms of degree four, three and two respectively gives the following equalities:

$$
\begin{gathered}
\rho=1 /\left(a_{1}+b_{1}\right), \alpha=\beta=0, \gamma=-m_{1}, \\
\alpha^{\prime}=2 \ell_{0}, \beta^{\prime}=-2 \omega \ell_{1}, \gamma^{\prime}=\ell_{0}^{2}-\omega \ell_{1}^{2} .
\end{gathered}
$$

Conditions (9) imply that $\mathcal{C}_{2}$ is absolutely reducible as

$$
f_{2}\left(x_{0}, x_{1}\right)=\left(x_{0}-\epsilon x_{1}+\ell_{0}-\epsilon \ell_{1}\right)\left(x_{0}+\epsilon x_{1}+\ell_{0}+\epsilon \ell_{1}\right) .
$$

Therefore case (a) cannot occur. Furthermore we get

$$
\begin{aligned}
& \mathcal{C}_{1}: f_{1}\left(x_{0}, x_{1}\right)=\left(x_{0}-i x_{1}\right)\left(x_{0}-i^{q} x_{1}\right)-m_{1}=0 \\
& \mathcal{C}_{2}: f_{2}\left(x_{0}, x_{1}\right)=\left(x_{0}-\epsilon x_{1}\right)\left(x_{0}+\epsilon x_{1}\right)+2 \ell_{0} x_{0}-2 \omega \ell_{1} x_{1}+\ell_{0}^{2}-\omega \ell_{1}^{2}=0 .
\end{aligned}
$$

Now, comparing the terms of degree one in (7) we get

$$
2 \ell_{0}\left(-m_{1}\right)=\ell_{1} m_{0}-m_{1} \ell_{0}-n_{1}
$$

and

$$
-2 \omega \ell_{1}\left(-m_{1}\right)=\omega \ell_{1} m_{1}-\ell_{0} m_{0}+n_{0}
$$

that is,

$$
\left\{\begin{array}{l}
m_{1} \ell_{0}+m_{0} \ell_{1}-n_{1}=0 \\
\omega m_{1} \ell_{1}+\ell_{0} m_{0}-n_{0}=0
\end{array} .\right.
$$

Conditions (10) give $\ell m-n=0$, which is impossible. Hence neither case (b) nor case (c) can occur.

Next suppose that $\Phi$ splits into two conjugate conics $\mathcal{C}_{1}$ and $\mathcal{C}_{2}$ over $\operatorname{GF}(q)$ with

$$
\mathcal{C}_{1}: c_{1}\left(x_{0}, x_{1}\right)=\left(x_{0}-\epsilon x_{1}\right)\left(x_{0}-i^{q} x_{1}\right)+\alpha x_{0}+\beta x_{1}+\gamma=0
$$

and

$$
\mathcal{C}_{2}: c_{2}\left(x_{0}, x_{1}\right)=\left(x_{0}+\epsilon x_{1}\right)\left(x_{0}-i x_{1}\right)+\alpha^{q} x_{0}+\beta^{q} x_{1}+\gamma^{q}=0,
$$

where $\alpha, \beta, \gamma \in \operatorname{GF}\left(q^{2}\right)$. If condition

$$
\rho f\left(x_{0}, x_{1}\right)=c_{1}\left(x_{0}, x_{1}\right) c_{2}\left(x_{0}, x_{1}\right)
$$

occurs, then comparing the terms of degree four we have $\rho=1 /\left(a_{1}+b_{1}\right)$, whereas comparing the terms of degree three we get

$$
\begin{aligned}
\left(x_{0}-i^{q} x_{1}\right)\left(x_{0}-i x_{1}\right)\left(2 \ell_{0} x_{0}-2 \omega \ell_{1} x_{1}\right)= & \left(x_{0}-\epsilon x_{1}\right)\left(x_{0}-i^{q} x_{1}\right)\left(\alpha^{q} x_{0}+\beta^{q} x_{1}\right) . \\
& +\left(x_{0}+\epsilon x_{1}\right)\left(x_{0}-i x_{1}\right)\left(\alpha x_{0}+\beta x_{1}\right)
\end{aligned} .
$$

Therefore $\left(x_{0}-i x_{1}\right)$ divides $\left(x_{0}-\epsilon x_{1}\right)\left(x_{0}-i^{q} x_{1}\right)\left(\alpha^{q} x_{0}+\beta^{q} x_{1}\right)$ and $\left(x_{0}-i^{q} x_{1}\right)$ divides $\left(x_{0}+\epsilon x_{1}\right)\left(x_{0}-i x_{1}\right)\left(\alpha x_{0}+\beta x_{1}\right)$. 
Since $i \neq i^{q}$, this leaves only few possibilities: either $i=\epsilon$ and hence $a_{0}=a_{1}=0$, or $\alpha x_{0}+\beta x_{1}=\alpha\left(x_{0}-i^{q} x_{1}\right)$. Since $a \neq 0$ we get:

$$
\mathcal{C}_{1}: c_{1}\left(x_{0}, x_{1}\right)=\left(x_{0}-\epsilon x_{1}\right)\left(x_{0}-i^{q} x_{1}\right)+\alpha\left(x_{0}-i^{q} x_{1}\right)+\gamma=0
$$

and

$$
\mathcal{C}_{2}: c_{2}\left(x_{0}, x_{1}\right)=\left(x_{0}+\epsilon x_{1}\right)\left(x_{0}-i x_{1}\right)+\alpha^{q}\left(x_{0}-i x_{1}\right)+\gamma^{q}=0,
$$

with $\alpha, \gamma \in \operatorname{GF}\left(q^{2}\right)$.

Moreover, (12) yields

$$
\left(2 \ell_{0} x_{0}-2 \omega \ell_{1} x_{1}\right)=\alpha^{q}\left(x_{0}-\epsilon x_{1}\right)+\alpha\left(x_{0}+\epsilon x_{1}\right)
$$

whence

$$
2 \ell_{0}=\alpha+\alpha^{q}, \quad-2 \omega \ell_{1} \frac{1}{\epsilon}=\left(\alpha-\alpha^{q}\right) ;
$$

then by considering the sum of the two last equations

$$
\alpha=\ell_{0}-\omega \ell_{1} \frac{1}{\epsilon}
$$

Then

$$
\mathcal{C}_{1}: c_{1}\left(x_{0}, x_{1}\right)=\left(x_{0}-\epsilon x_{1}\right)\left(x_{0}-i^{q} x_{1}\right)+\left(\ell_{0}-\frac{\omega}{\epsilon} \ell_{1}\right)\left(x_{0}-i^{q} x_{1}\right)+\gamma=0
$$

and

$$
\mathcal{C}_{2}: c_{2}\left(x_{0}, x_{1}\right)=\left(x_{0}+\epsilon x_{1}\right)\left(x_{0}-i x_{1}\right)+\left(\ell_{0}+\frac{\omega}{\epsilon} \ell_{1}\right)\left(x_{0}-i x_{1}\right)+\gamma^{q}=0,
$$

with $\gamma \in \operatorname{GF}\left(q^{2}\right)$. By considering the degree two terms in (11) we get

$$
\begin{aligned}
& \left(x_{0}-i x_{1}\right)\left(x_{0}-i^{q} x_{1}\right)\left(\ell_{0}^{2}-\omega \ell_{1}^{2}\right)-\rho m_{1}\left(x_{0}-\epsilon x_{1}\right)\left(x_{0}+\epsilon x_{1}\right)= \\
& \gamma^{q}\left(x_{0}-\epsilon x_{1}\right)\left(x_{0}-i^{q} x_{1}\right)+\gamma\left(x_{0}+\epsilon x_{1}\right)\left(x_{0}-i x_{1}\right)+\left(\ell_{0}^{2}-\omega \ell_{1}^{2}\right)\left(x_{0}-i^{q} x_{1}\right)\left(x_{0}-i x_{1}\right)
\end{aligned}
$$

that is,

$$
-\rho m_{1}\left(x_{0}-\epsilon x_{1}\right)\left(x_{0}+\epsilon x_{1}\right)=\gamma^{q}\left(x_{0}-\epsilon x_{1}\right)\left(x_{0}-i^{q} x_{1}\right)+\gamma\left(x_{0}+\epsilon x_{1}\right)\left(x_{0}-i x_{1}\right) .
$$

Arguing as before, we have that $\left(x_{0}-\epsilon x_{1}\right)$ divides $\left(x_{0}-i x_{1}\right)$. Hence we get $a_{0}=a_{1}=0$ unless $m_{1}=0$. But if $m_{1}=0$ then

$$
\gamma^{q}\left(x_{0}-\epsilon x_{1}\right)\left(x_{0}-i^{q} x_{1}\right)=-\gamma\left(x_{0}+\epsilon x_{1}\right)\left(x_{0}-i x_{1}\right),
$$

whence $\gamma^{q}=\gamma=0$. Therefore all the coefficients in $f$ of degree less than two must be equal to zero, that is $\left(\rho\left(\ell_{1} m_{0}-n_{1}\right), \rho\left(-\ell_{0} m_{0}+n_{0}\right), \rho\left(-\ell_{0} n_{1}+\ell_{1} n_{0}\right)\right)=(0,0,0)$, and this gives $\ell m-n=0$, a contradiction.

Now consider the even $q$ case. We may assume that $\mathcal{C}_{1}$ and $\mathcal{C}_{2}$ have equations

$$
\begin{aligned}
& \mathcal{C}_{1}: f_{1}\left(x_{0}, x_{1}\right)=\left(x_{0}-i x_{1}\right)\left(x_{0}-i^{q} x_{1}\right)+\alpha x_{0}+\beta x_{1}+\gamma=0 \\
& \mathcal{C}_{2}: f_{2}\left(x_{0}, x_{1}\right)=\left(x_{0}-\epsilon x_{1}\right)\left(x_{0}-(1+\epsilon) x_{1}\right)+\alpha^{\prime} x_{0}+\beta^{\prime} x_{1}+\gamma^{\prime}=0
\end{aligned}
$$


where $\alpha, \alpha^{\prime}, \beta, \beta^{\prime}, \gamma, \gamma^{\prime} \in \mathrm{GF}(q)$ and

$$
\rho f\left(x_{0}, x_{1}\right)=f_{1}\left(x_{0}, x_{1}\right) f_{2}\left(x_{0}, x_{1}\right) .
$$

Arguing as in the odd $q$ case we have the following equalities:

$$
\begin{array}{r}
\rho=1 /\left(a_{1}+b_{1}\right) \alpha=\beta=0, \gamma=m_{1}, \\
\alpha^{\prime}=\ell_{1}, \beta^{\prime}=\ell_{0}, \gamma^{\prime}=\ell_{0}^{2}+\ell_{0} \ell_{1}+\nu \ell_{1}^{2},
\end{array}
$$

that come by comparing in (13) the terms of degree four, three and two.

Conditions (15) imply that $\mathcal{C}_{2}$ is absolutely reducible as

$$
f_{2}\left(x_{0}, x_{1}\right)=\left(x_{0}-\epsilon x_{1}+\ell_{0}-\epsilon \ell_{1}\right)\left[x_{0}-(1+\epsilon) x_{1}+\ell_{0}-(1+\epsilon) \ell_{1}\right] .
$$

Therefore case (a) cannot occur. Then we have

$$
\begin{aligned}
& \mathcal{C}_{1}: f_{1}\left(x_{0}, x_{1}\right)=\left(x_{0}-i x_{1}\right)\left(x_{0}-i^{q} x_{1}\right)+m_{1}=0, \\
& \mathcal{C}_{2}: f_{2}\left(x_{0}, x_{1}\right)=\left(x_{0}-\epsilon x_{1}\right)\left(x_{0}-(1+\epsilon) x_{1}\right)+\ell_{1} x_{0}+\ell_{0} x_{1}+\ell_{0}^{2}+\ell_{0} \ell_{1}+\nu \ell_{1}^{2}=0 .
\end{aligned}
$$

By considering the degree one terms in (13) we get

$$
\ell_{0} m_{1}=\ell_{0} m_{0}+\ell_{0} m_{1}+\nu \ell_{1} m_{1}+n_{0}
$$

and

$$
\ell_{1} m_{1}=\ell_{0} m_{1}+\ell_{1} m_{0}+n_{1}
$$

that is,

$$
\left\{\begin{array}{l}
\ell_{0} m_{0}+\nu \ell_{1} m_{1}+n_{0}=0 \\
\ell_{1} m_{1}+\ell_{0} m_{1}+\ell_{1} m_{0}+n_{1}=0
\end{array} .\right.
$$

Conditions (16) give $\ell m-n=0$, which is impossible. Hence neither case (b) nor case (c) can occur.

Now, suppose that $\Phi$ splits into two conjugate conics $\mathcal{C}_{1}$ and $\mathcal{C}_{2}$ over $\operatorname{GF}(q)$ with

$$
\mathcal{C}_{1}: c_{1}\left(x_{0}, x_{1}\right)=\left(x_{0}-\epsilon x_{1}\right)\left(x_{0}-i x_{1}\right)+\alpha x_{0}+\beta x_{1}+\gamma=0
$$

and

$$
\mathcal{C}_{2}: c_{2}\left(x_{0}, x_{1}\right)=\left(x_{0}-(1+\epsilon) x_{1}\right)\left(x_{0}-i^{q} x_{1}\right)+\alpha^{q} x_{0}+\beta^{q} x_{1}+\gamma^{q}=0,
$$

with $\alpha, \beta, \gamma \in \operatorname{GF}\left(q^{2}\right)$. If condition

$$
\rho f\left(x_{0}, x_{1}\right)=c_{1}\left(x_{0}, x_{1}\right) c_{2}\left(x_{0}, x_{1}\right)
$$

occurs, then comparing the terms of degree four we have $\rho=1 /\left(a_{1}+b_{1}\right)$, whereas comparing the terms of degree three we get

$$
\begin{aligned}
\left(x_{0}-i x_{1}\right)\left(x_{0}-i^{q} x_{1}\right)\left(\ell_{0} x_{1}+\ell_{1} x_{0}\right) & =\left(x_{0}-\epsilon x_{1}\right)\left(x_{0}-i x_{1}\right)\left(\alpha^{q} x_{0}+\beta^{q} x_{1}\right)+ \\
& \left(x_{0}-(1+\epsilon) x_{1}\right)\left(x_{0}-i^{q} x_{1}\right)\left(\alpha x_{0}+\beta x_{1}\right) .
\end{aligned}
$$


Therefore $\left(x_{0}-i x_{1}\right)$ divides $\left(\alpha x_{0}+\beta x_{1}\right)$. Since $\left(a_{0}, a_{1}\right) \neq(0,0)$, this leaves $\alpha x_{0}+\beta x_{1}=$ $\alpha\left(x_{0}-i x_{1}\right)$ Moreover, (18) yields

$$
\left(\ell_{0} x_{1}+\ell_{1} x_{0}\right)=\alpha^{q}\left(x_{0}-\epsilon x_{1}\right)+\alpha\left(x_{0}-(1+\epsilon) x_{1}\right)
$$

whence

$$
\ell_{1}=\alpha+\alpha^{q}, \quad-(1+\epsilon) \alpha-\epsilon \alpha^{q}=\ell_{0}
$$

then we get

$$
\alpha=\ell_{0}+\epsilon \ell_{1} .
$$

Hence

$$
\mathcal{C}_{1}: c_{1}\left(x_{0}, x_{1}\right)=\left(x_{0}-\epsilon x_{1}\right)\left(x_{0}-i x_{1}\right)+\left(\ell_{0}+\epsilon \ell_{1}\right)\left(x_{0}-i x_{1}\right)+\gamma=0
$$

and

$$
\mathcal{C}_{2}: c_{2}\left(x_{0}, x_{1}\right)=\left(x_{0}-(1+\epsilon) x_{1}\right)\left(x_{0}-i^{q} x_{1}\right)+\left(\ell_{0}+(1+\epsilon) \ell_{1}\right)\left(x_{0}-i^{q} x_{1}\right)+\gamma^{q}=0,
$$

with $\gamma \in \operatorname{GF}\left(q^{2}\right)$.

By considering the degree two terms in (17) we get

$$
\rho m_{1}\left(x_{0}-\epsilon x_{1}\right)\left(x_{0}-(1+\epsilon) x_{1}\right)=\gamma^{q}\left(x_{0}-\epsilon x_{1}\right)\left(x_{0}-i x_{1}\right)+\gamma\left(x_{0}-(1+\epsilon) x_{1}\right)\left(x_{0}-i^{q} x_{1}\right) .
$$

Arguing as before, we get either that $\left(x_{0}-\epsilon x_{1}\right)$ divides $\left(x_{0}-i^{q} x_{1}\right)$, which is impossible, or $m_{1}=0$. But if $m_{1}=0$ then

$$
\gamma^{q}\left(x_{0}-\epsilon x_{1}\right)\left(x_{0}-i x_{1}\right)=\gamma\left(x_{0}-(1+\epsilon) x_{1}\right)\left(x_{0}-i^{q} x_{1}\right),
$$

whence $\gamma^{q}=\gamma=0$. Hence all the coefficients in $f$ of degree less than two must be equal to zero giving $\ell m-n=0$, a contradiction.

Now, we can apply Hasse's Theorem to the irreducible curve $\Phi$; see [8, Theorem 9.57]. It follows that

$$
q+1-6 \sqrt{q} \leqslant R_{q} \leqslant q+1+6 \sqrt{q},
$$

where $R_{q}$ is the number of points of $\Phi$ which lie in $\operatorname{PG}(2, q)$. Therefore, $\mathcal{U}_{a, b} \cap \Omega$ is a $k$-arc with $q-6 \sqrt{q}-2 \leqslant k \leqslant q+6 \sqrt{q}-2$ because $\Phi$ has four points at infinity which do not correspond to any points of $\Omega$. Since an absolutely irreducible quartic and a conic share at most eight points, $\mathcal{U}_{a, b} \cap \Omega$ meets every subconic of $\Omega$ in at most eight points.

\section{References}

[1] G. Donati, N. Durante, G. Korchmàros, On the intersection pattern of a unital and an oval in $\mathrm{PG}\left(2, q^{2}\right)$, Finite Fields Appl. 15 (2009), 785-795.

[2] S.G. Barwick and G.L. Ebert, Unitals in projective planes. Springer Monographs in Mathematics. Springer, New York, 2008. 
[3] R.D. Baker and G.L. Ebert, On Buekenhout-Metz unitals of odd order, J. Combin. Theory Ser. A 60 (1992) 67-84.

[4] G.L. Ebert, On Buekenhout-Metz unitals of even order, European J. Combin. 13 (1992) 109-117.

[5] G.L. Ebert, Hermitian Arcs, Rend. Circ. Mat. Palermo (2) Suppl. 51 (1998) 87-105.

[6] G.L. Ebert, Buekenhout unitals, Discrete Math. 208/209 (1999) 247-260.

[7] J.W.P. Hirschfeld, Projective geometries over finite fields, Second edition. Oxford Mathematical Monographs. The Clarendon Press, Oxford University Press, New York, 1998.

[8] J.W.P. Hirschfeld, G. Korchmáros and F. Torres Algebraic Curves over a Finite Field, Princeton Series in Applied Mathematics. Princeton University Press, Princeton, NJ, 2008. 